\title{
Gavroche et ses pairs : aspects de la violence politique du groupe enfantin en France au XIXe siècle
}

\section{Frédéric Chauvaud}

\section{(2) OpenEdition Journals}

Édition électronique

URL : http://journals.openedition.org/conflits/463

DOI : $10.4000 /$ conflits.463

ISSN : 1777-5345

Éditeur :

CCLS - Centre d'études sur les conflits lilberté et sécurité, L'Harmattan

Édition imprimée

Date de publication : 15 juillet 1995

ISSN : 1157-996X

Référence électronique

Frédéric Chauvaud, «Gavroche et ses pairs : aspects de la violence politique du groupe enfantin en France au XIXe siècle », Cultures \& Conflits [En ligne], 18 | été 1995, mis en ligne le 04 mars 2005, consulté le 30 mars 2021. URL : http://journals.openedition.org/conflits/463 ; DOI : https://doi.org/ $10.4000 /$ conflits.463

Ce document a été généré automatiquement le 30 mars 2021.

Creative Commons License 


\title{
Gavroche et ses pairs : aspects de la violence politique du groupe enfantin en France au XIXe siècle
}

\author{
Frédéric Chauvaud
}

1 «Moineau becquetant ", « nain invulnérable de la mêlée ", "étrange gamin fée » sont quelques-unes des métaphores déployées par Victor Hugo en 1862 pour camper le personnage de Gavroche ${ }^{1}$. Le martyre du fils des Thénardier, couché sur le pavé par les tirs des lignards, imposera l'image de l'enfant pris dans les rets de la violence politique. C'est au cours d'un entre-deux tumultueux, qui progresse des premières années de la Monarchie de Juillet jusqu'à l'enracinement de la IIIe République, que surgit la figure novatrice du gamin des barricades. Pourtant, pas plus que le sentiment de l'enfance n'a été inventé au XIXe siècle, les enfants terribles, selon le lexicographe du temps, ne sont «un produit spécial à notre époque " ${ }^{2}$. Alors, des interrogations naissent de la confusion attachée au statut de l'enfance comme à la vacuité de l'analyse ${ }^{3}$. En effet, le domaine de l'enfance politique au XIXe siècle est une contrée vierge. L'indigence des études suffit à le souligner. Tout au plus s'est-on attaché à l'enfance pitoyable, victime de leur statut et du vide de la législation sociale ${ }^{4}$; à l'enfance ouvrière ${ }^{5}$ considérée comme coupable ${ }^{6}$, car composée de voleurs et de vagabonds; à l'enfance punissable, emplissant à la fin du grand XIXe siècle seulement, les " tribunaux pour enfants ${ }^{7}$; ou encore à l'enfance considérée en tant qu'agent des passions françaises ${ }^{8}$. Quant à la marmaille turbulente, elle est devenue sujet d'enquête à partir du moment où il fallait en faire l'éducation ${ }^{9}$ ou qu'il fallait la scolariser. Mais « la clientèle forcée » de l'école a moins retenue l'attention que le groupe des maîtres. Toutefois, dans une analyse fulgurante mais trop brève, Maurice Crubellier analyse la société enfantine de l'école élémentaire comme un groupe produisant une "contre-culture». Rites clandestins, rôles secrets, jeux violents sont l'apanage des enfants en dehors de la surveillance des adultes ${ }^{10}$. Mais de cette floraison, la politique est absente. Reste donc Gavroche, par qui il faut bien commencer. Comment la représentation du massacre d'un jeune garçon, à laquelle toute violence juvénile fait encore aujourd'hui référence, s'est-elle imposée? De quelle manière, au-delà des apparences discursives, dans l'épaisseur des massacres 
et de la guerre civile, a-t-elle été forgée? Les explications risquent de rester fragmentaires puisqu'on chercherait en vain à saisir tous les segments de la mémoire d'une époque révolue. Toutefois, l'historien peut user de détours, se montrer soucieux de décomposer les phases d'une singulière alchimie, s'attacher à suivre l'opération occulte visant à transmuter un type littéraire en mythe politique qui révélera l'existence d'une violence politique des enfants.

2 La construction d'un archétype Au XIXe siècle, l'enfant semble exercer une fascination distante. On rencontre aussi bien l'être gracieux et conventionnel, le malheureux abandonné dans les cloaques putrides que les avatars de l'enfant sauvage. On discerne bien, dans nombre de domaines, une multiplication des indices: l'enfant maltraité, tyrannisé et molesté allant du Petit Chose à Poil de Carotte, emplit timidement la littérature; les enquêteurs sociaux, arpentant les strates de la société, découvrent le travail des enfants et donnent des descriptions hallucinées qui pousseront le législateur à intervenir; à la fin des années 1870, tandis que la criminologie prend son essor et invente le "criminel-né », l'enfant suscite un regain d'intérêt, il est surveillé avec plus d'intensité, car dès sa naissance il représente une menace virtuelle. Mais, c'est Victor Hugo qui impose en 1853 la vision de l'enfant mort, victime expiatoire de la violence politique; puis, dix ans plus tard, il donne le spectacle $\mathrm{du}$ massacre de Gavroche. Dans la première version, mise en scène dans Les Châtiments, un jeune garçon est fauché par "deux balles dans la tête", tirées par les "prétoriens» du prince-président. Situé au début du recueil (livre deuxième), "Souvenir de la nuit du quatre», est un cri dénonçant l'ignominie du prince, responsable d'avoir tué un enfant de sept ans. Or le personnage de papier possède au moins un modèle de chair que Victor Hugo a rencontré en 1851. Le 4 décembre, une soixantaine de barricades, derrière lesquelles se retranchent 1200 défenseurs sont hâtivement érigées. La troupe, laissée volontairement à l'écart depuis le début du coup d'Etat, porte l'assaut. Mais sur les boulevards, tandis que les curieux s'agglutinent sur les trottoirs et les balcons, le sang froid déserte la soldatesque qui « criblant de balles ces ennemis imaginaires " ${ }^{11}$ laisse des dizaines de morts et des centaines de blessés; parmi les fusillés, des enfants. La pâle silhouette ensanglantée de l'un d'eux sert donc de référence obligée à Victor Hugo, c'est son image qui court, dans le recueil de l'exil, de Nox à Lux. Le public pourtant n'en fit pas une figure emblématique. La seconde version n'est autre que la fiction romanesque des Misérables, dont les combats de rue ont été transposés en 1832, pendant les journées insurrectionnelles des 5 et 6 juin. C'est le souvenir de l'enfant Boursier, abattu comme des dizaines de passants en 1851, qui inspire le personnage de Gavroche. Ainsi, le 15 juin 1862, lorsque le roman s'achève, il devient la première véritable oeuvre d'importance, écrite en français, qui traite des enfants, thème redoublé par la violence politique, la mitraille et la mort. Et, signe des temps, la même année, tandis que l'Empire traverse les « années incertaines " ${ }^{12}$, dans le Paris d'Hausmann une "maquette", grandeur nature figure le futur boulevard du Prince-Eugène, difficile à « barrer » par une unique barricade. La réception de l'oeuvre échappe aussi bien à l'auteur qu'à la censure. L'intrication du réel et de la fiction a certes suscité bien des alarmes et de virulentes polémiques, mais le personnage de Gavroche, empruntant de multiples canaux, s'ancre presque d'emblée dans la mémoire et se mue en archétype. La dilatation romanesque est ici exemplaire. En avril 1871, la Commune de Paris connaît ses premières dissensions. Sur la sellette Raoul Rigault qui, pris à partie, doit adopter un «système " afin de se défendre. Pour parer les reproches, il se contente de "hausser les épaules avec son geste de gavroche" ${ }^{13}$. En mai, la 
Commune est écrasée; tandis que les prisonniers sont parqués et que les cadavres jonchent les rues, les Versaillais découvrent une réalité incongrue : des enfants par centaines, assombris et désoeuvrés, sont livrés à eux-mêmes. La Patrie, l'un des quelques journaux qui ne fut pas interdit lors du coup d'Etat de décembre 1851, les qualifiera de "gavroches » ou de «pâles voyous » ${ }^{14}$, revisitant ainsi les métaphores du proscrit le plus célèbre du Second Empire. Il convient de souligner ici que l'image du garçon des barricades, d'abord polymorphe, a mis quelques années avant de se cristalliser en une représentation unanime. Ainsi, dans le Grand Dictionnaire universel de Pierre Larousse, Gavroche, dans un premier temps, est accueilli et intégré au patrimoine républicain avec réserve. Au lendemain de la Commune, en 1872, l'article "Gavroche », lapidaire et sec, indique que l'expression désigne le gamin de Paris, mais, ajoute le lexicographe, elle possède alors une connotation vulgaire. Deux ans plus tard, lors de la livraison du onzième tome, c'est une toute autre version qui est donnée dans un compte rendu dithyrambique des Misérables. On peut lire que Gavroche donne l'occasion d'une "des plus pénétrantes études physiologiques» ${ }^{15}$. Désormais, la profusion des images s'évanouit et seul survit dans la mémoire collective le fait que "Gavroche aussi meurt héroïquement, en vrai gamin de Paris ». Ainsi s'achève un cycle. Le type littéraire, fondé à l'origine par l'observation directe, s'extirpe du roman pour aller à la rencontre d'une sensibilité collégiale et enraciner un mythe politique.

3 Scrutant l'imaginaire politique, Raoul Girardet, dans un travail pionnier, propose de conduire une lecture serrée de l'histoire idéologique à partir de quatre "grands ensembles mythologiques » : la Conspiration, l'Age d'or, le Sauveur, l'Unité ${ }^{16}$. Mais à cette liste, qui ne prétend aucunement à l'exhaustivité, il conviendrait d'accoler le thème du martyre, dont Gavroche constitue une des composantes essentielles. Pour simplifier, on peut hasarder que le mythe se lit comme un récit, possède une cohérence et est porteur d'un message qui lui donne une fonction. Gavroche détient tout cela. Courageux, gouailleur, généreux, indépendant, autant de traits suggérant avec insistance qu'il appartient à une société juvénile, adhérant sans l'ombre d'une hésitation à une configuration de valeurs romantiques qu'il met au service du projet républicain des adultes, sans pour autant être manoeuvré par eux. De sorte que, échappant aux influences sournoises et aux pressions plus directes, Gavroche fait le sacrifice volontaire de sa vie. La République officielle devra composer avec le mythe. Personnage de roman, il ne deviendra pas un héros de l'histoire de France, mais un héros du coeur. A Lyon, à Rouen, à Marseille, à Montmartre, à Belleville... nul doute que dans l'espace intérieur des déshérités et de ceux qui rêvent à un autre monde, il occupe une place de choix. Plus tard, avec le retrait de la violence et sa domestication, le mythe aura tendance à s'édulcorer, Gavroche, deviendra le titi parisien, plus proche des enfants des rues croqués par Poulbot, que des victimes enfantines des grandes «collisions». A moins, peut-être, qu'il ne se métamorphose en "apache » ${ }^{17}$ qu'il $^{\prime}$ faudrait fouetter?

4 La consistance du réel Selon Philippe Ariès, à l'orée de la Révolution de 1789, l'adolescence et l'enfance s'associent dans une même identité à tel point qu'il n'existe pas véritablement de mots pour les désigner et les distinguer. Mais, ajoute-t-il, concluant une étude liminaire: "tout se passe comme si, à chaque époque, correspondait un âge privilégié et une périodisation particulière de la vie humaine : la « jeunesse est l'âge privilégié du XVIIe siècle, l'enfance du XIXe siècle, l'adolescence du XXe " ${ }^{18}$. Or dès que l'on traite de l'enfance, on se met presque aussitôt à morceler cet âge de la vie, inventant la petite enfance et l'âge de raison, commençant à sept ans et 
dont sont particulièrement friands les manuels de savoir vivre et d'éducation destinés aux jeunes mères. D'aucuns prétendent que l'enfant est l'être que l'on peut porter dans ses bras, donnant ainsi une prime à l'enfant malingre ou étique. D'autres, comme les rédacteurs de la Gazette des tribunaux, discernent des enfants de seize ans, voire de dix sept. Dans les sociétés rurales, l'enfant est celui qui n'est pas encore établi ou marié, qui effectue une activité un peu marginale, vacher ou gardien d'oies. Dans le coeur des cités ou à proximité des barrières existent, disent les rapports de gendarmerie, des bandes d'enfants. Mais ce peuple enfantin, parfois suspecté de sombres turpitudes, n'attire qu'en de très rares occasions l'intérêt des autorités chargées de contrôler l'esprit public. Même, le préfet Gisquet ne songerait à les associer à une quelconque activité politique. Reste que, pour Georges Weill, la renaissance de l'idée républicaine sous la Restauration est due à la jeunesse, mais il s'agit de la jeunesse des écoles, gagnée à la cause de la réforme, se métamorphosant en générations romantiques. Les enfants, eux, tenus à l'écart du renouveau, ne sont pourtant pas exclus de tout apprentissage politique. Dans la famille, ou dans un petit cercle de proches, les récits enflammés et les souvenirs vivaces participent à l'éducation politique. C'est ainsi que Cavaignac et Carnot recueillent l'enseignement de leurs pères; Jean Reynaud celui de Merlin de Thionville ${ }^{19}$. Mais des différents gradins sociaux formant la société, nul enfant ne participe directement aux violences politiques. Toutefois, peu de temps après 1830 et l'avènement de la monarchie nouvelle, la figure emblématique de Gavroche, du moins celle de l'un de ses devanciers, surgit par intermittence. Il suffit de songer à la Liberté sur les barricades de Delacroix. En 1831, tout d'abord à Lyon. Au mois de novembre, les fabricants ne respectent pas le tarif, les Canuts, c'est-à-dire les chefs d'atelier et les ouvriers décident de faire arrêter les métiers. L'insurrection commence le 21. Des contemporains ont noté la détermination et le courage des enfants. Ce sont eux qui forment les barricades. Ils ne se contentent pas d'édifier des entraves à l'intérieur de la cité : "Ces petits malheureux ont montré la plus grande insouciance du danger et quelquefois un mépris complet de la vie $"{ }^{20}$. Souligner, même de façon fragmentaire leur participation peut répondre à une vision instrumentale. Pour le chroniqueur, il s'agirait de montrer l'adhésion totale, au delà des différences de condition, de sexe et de classes d'âge, autour d'aspirations communes. La posture de celui qui parle montre qu'il ne s'agit pas d'une préoccupation majeure. Observateur exigeant de ces journées tourmentées, il note ce qui le frappe : le concours et le dévouement des enfants qui ressortissent d'une transgression de "l'ordre du monde". Sujet minuscule et insaisissable, la trace des enfants est grossie lors des grandes violences collectives. Il est alors plus facile d'en saisir les manifestations, plus particulièrement en 1832 et en 1848. Tandis que sévit le choléra morbus, le convoi funèbre du général Lamarque serpente dans les rues de Paris. Le 5 juin 1832, la parade funèbre dérape, des barricades sont dressées rues Saint-Méry, Aubry-le-Boucher et Saint-Martin. Des enfants, confectionnant des « bourres » se saisissent parfois des fusils pour suppléer au manque de combattants. Un enfant de douze ans est ainsi blessé à la tête, et on « ne put obtenir de lui qu'il quittât la barricade ${ }^{21}$. Derrière, la barricade de Saint-Merri, deux autres gamins, poussés par la curiosité, se bousculent afin de mieux voir la scène, quitte à exposer un peu trop longuement un visage. Le 25 février 1848 Victor Hugo sort de l'Hôtel de ville où siège le Gouvernement provisoire, il passe ensuite devant la caserne des Minimes. Dehors, il découvre une sentinelle en faction. L'ancien pair de France la reconnaît pour l'avoir aperçue 17 heures plus tôt: «un garçonnet d'une quinzaine d'années, armé d'un grand fusil de la ligne » ${ }^{22}$. Au mois de mars et d'avril, d'autres 
enfants, plus jeunes encore, réceptacles malléables des événements, miment le comportement des adultes ou des frères aînés. L'un, âgé de sept ans, lance sa casquette après chaque refrain d'une chanson dans laquelle il est question de guillotine et de vengeance, de Louis Philippe et de Guizot; un deuxième, " un marmot de trois ans ", s'exerce aussi à la chanson et fait retentir Mourir pour la patrie ${ }^{23}$. En juin 1848, cette fois dans la capitale phocéenne, tandis que les ouvriers prennent le 22 le parti de la sédition, une colonne d'attaque se lance vers la barricade qui fermait la « Grand'rue du côté du palais de justice ", là, on vit paraître, rapporte un des assaillants "la tête d'un enfant qui disparut bientôt " ${ }^{24}$. Chaque être enfantin ne peut être réduit à un comportement unique. Il n'en reste pas moins que l'identité des enfants reste, au cours du premier grand XIXe siècle, brouillée. Schématiquement, jusqu'aux années 1880, époque à laquelle on se persuadera de leur cruauté naturelle, puis à laquelle on cherchera à remédier à ses « instincts anti-sociaux innés » ${ }^{25}$, ceux qui se penchent sur l'amour filial ou sur l'éducation des enfants appartiennent à un entre-deux de l'observation. Les diverses disciplines demeurent balbutiantes en la matière, elles commencent tout juste à peindre et à décrire les enfants. Il n'en reste pas moins que chaque fois qu'un acte brutal s'apparente à une action politique, il surprend et il provoque presque aussitôt la désapprobation. Mais il y a pis, l'autonomie du groupe des enfants suscite l'effroi car elle ruine alors l'autorité parentale et la représentation du rôle des individus. Tout se passe comme si une coupure venait d'être faite dans le lien social. On sait que la violence juvénile sous la Restauration, tolérée à condition de s'exercer dans les campagnes, se déployait selon un système d'appartenance communautaire. Parfois les jeunes garçons étaient associés à ces rixes qui pouvaient déraper vers une violence extrême. En rase campagne ou à la lisière des villes, le compagnonnage a favorisé auprès de jeunes apprentis une culture de la violence, mais celle-ci est davantage rite identitaire que manifestation politique. Cependant, dans le coeur de la "grande cité ", un fait divers traité par La Gazette des tribunaux acquiert un caractère emblématique car en filigrane il dévoile la surprise et l'inquiétude des adultes face à des enfants agissant comme un groupe indépendant. Cette "petite insurrection buissonnière ", dont l'origine reste obscure, mais dont la logique seule importe ici, s'est brusquement imposée le 31 janvier 1840, vers 10 heures du soir. Dans l'intérieur du jardin du palais-Royal, une "centaine d'enfants » s'en prennent à un surveillant. Un sergent de ville flanqué d'un garde municipal sont diligentés par le commissaire de police du quartier. A leur vue « l'émeute des enfants » redouble de furie et « ce ne fut qu'en tirant le sabre qu'ils purent parvenir à dégager le gardien » ${ }^{26}$. Mais, au-delà des apparences et des représentations, rien de plus frappant que la place des enfants pendant la Commune de Paris. L'extrême matérialité de la violence politique fait surgir un monde enfantin, prisonnier du temps présent, ballotté entre les plus folles espérances et l'abattement le plus poignant.

5 L'instantané de la " guerre des barricades » 1871 encourage le « ruissellement » des configurations de la violence politique. En premier lieu s'impose la pratique du «massacre des innocents». Lors de la guerre des rues, les corps des enfants prennent place dans les "dépôts de cadavres ", auprès des dépouilles mortelles des adultes. Une telle présence est attestée à plusieurs reprises, tout se passe pourtant comme si elle échappait en partie au toucher du regard. En 1851 et en 1871, quelques témoignages parcimonieux, vite resserrés dans des cartons d'archives, en font une mention hâtive. Variation macabre sur le thème, on rapporte que le 26 mai 1871, chez le restaurateur Deffieux, entre les théâtres de la porte Saint-Martin et de l'ambiguë, plusieurs enfants 
auraient été tués, les traces du massacre auraient disparu avec l'incendie ${ }^{27}$. Quelques jours auparavant, après l'assaut très violent de la barricade de la rue Lepic, près de la place Pigalle quelques cadavres relevés derrière les vestiges de la malheureuse digue, sont tournés contre le sol, parmi eux un "pauvre enfant" ${ }^{28}$. A proximité de la pointe Saint-Eustache, d'intenses combats fauchent les Communards, des enfants les remplacent, ils ne sont pas épargnés par le massacre ${ }^{29}$. Victorine B, camouflée en gamin échappe au massacre, mais à Belleville, sur la place de la mairie, « sur une pile de morts il y avait une pauvre petite fillette qui pouvait avoir dans les huit ans, jolie, aux cheveux blonds bouclés ", un soldat de ligne avait eu une «monstrueuse idée » de mise en scène ${ }^{30}$. La répression sanglante encourage l'horreur, elle donne aussi l'occasion à quelques jeunes victimes de reprendre à leur compte un code de l'honneur que nombre d'adultes ont abandonné depuis longtemps. Ainsi, faubourg du temple, le 25 mai, le " plus enragé tireur est un enfant ». Après l'attaque, les prisonniers vont être fusillés, l'enfant veut porter sa montre à sa mère : «L'officier, involontairement ému le laisse partir, croyant bien ne plus le revoir. Trois minutes après, un «Me voilà !» C'est l'enfant qui saute sur le trottoir, et, lestement s'adosse au mur..." ${ }^{31}$. La Semaine sanglante, davantage que toute autre période, sollicite les enfants. Si leur sacrifice est exalté, c'est leur dévouement au service de la génération adulte qui est d'abord valorisé. Dès que les troupes de Versailles pénètrent dans Paris, par l'Est, le 21 mai, les enfants sont très nombreux à participer aux travaux de défense et d'édification. Le dernier numéro du «Journal officiel » de la Commune relate avec un optimisme factice que partout des barricades se sont élevées : «Les enfants, les femmes y ont travaillé toute la journée. Nous avons vu, dans une des rues transversales du faubourg du Temple un de ces ouvrages entièrement élevé en quelques heures par une vingtaines d'enfants dont le plus âgé n'avait pas quatorze ans " ${ }^{32}$. Une telle participation expose de nombreux enfants aux mouvements tournants, aux impacts des balles et aux déflagrations des obus. La défense du Paris insurgé mobilise toutes les énergies, car les jeunes bâtisseurs de barricades se mettent en scène devant leurs pairs et leurs aînés. D'aucuns prétendront que le fils participe et encourage la violence politique du père en lui préparant un rempart inexpugnable. Ainsi toutes les barricades de la rive gauche, du Pont Saint-Michel jusqu'en haut de l'observatoire, auraient été emplies et consolidées par « une masse de gamins travaillant à piocher la terre, et à la jeter sur les barricades en construction" ${ }^{33}$. L'élévation, la consolidation et la protection des barrages urbains entraînent de singuliers glissements. Le gamin passe ainsi du rôle de bâtisseur à celui de combattant. Alors face à sa famille, ses voisins, ses camarades, il s'expose inconsidérément. L'affrontement encourage donc un processus d'héroïsation. Il entre parfois dans ce sacrifice un aspect ludique, à la manière de Gavroche. Ainsi, Dauteille, enfant de 15 ans, quitte lestement la barricade du Château d'eau, ramasse sous le feu le képi d'un lieutenant tué et ramène son trophée à l'abri du tir des lignards 34. Mais la violence juvénile est parfois encadrée avec rigueur. En effet, un corps d'armée a été constitué pour les enfants : les Pupilles de la Commune. «Enrôlé de leur plein gré (...) ils ont été habillés, équipés et armés ; un seul d'entre eux prétend ne pas avoir reçu de fusil et s'est borné, dans son costume de pupille, à remplir des sacs de terre " ${ }^{35}$. Une telle structure borne l'expression de la violence juvénile tout en lui donnant la possibilité de se déployer pleinement lors des combats de rue. Des pupilles deviennent des supplétifs des ambulances, emportant les blessés sur des brancards; d'autres pupilles, à l'écart des adultes, ont recréé une micro-société hiérarchisée et défendent seuls la rue Magnan $^{36}$. Combien étaient-ils à participer aux combats? 
L'estimation chiffrée reste hasardeuse. Toutefois, la justice militaire se penchera sur le cas de 651 enfants : $36 \%$ sont âgés de 16 ans, $35 \%$ ont 15 ans, $16 \% 14$ ans, $7 \% 13$ ans et tous les autres ont entre 7 et 12 ans $^{37}$. Pendant les événements, les enfants sont ramassés et parqués, attendant d'être réclamés par leurs parents. Les petits prisonniers sont amenés à Versailles. La Patrie du 2 juin écrira qu'«il y a pour les dompter d'excellentes colonies agricoles toutes prêtes à leur donner l'hospitalité ». Le rédacteur précisera qu'ils "sont encore à l'état de cire». Reste enfin une dernière réalité à observer, totalement occultée. Dans les rangs des Versaillais se rencontrent aussi des gamins. Par exemple, un enfant de troupe de onze ans participe aux combats de rue ${ }^{38}$. Le journal Le Siècle relate qu'un des Communards fusillés "à la place du trône ", avait avec lui «ses deux petits enfants, âgés l'un de dix ans, l'autre de huit ans ». Le plus grand, immédiatement adopté par les lignards, est enrôlé à son tour dans les enfants de troupe, emmené dans le sillage des soldats et promu mascotte du régiment ${ }^{39}$. Au XIXe siècle, tandis que les représentations de la populace et de la foule se dissipent, les observateurs sociaux et les journalistes distinguent avec plus de netteté les différents acteurs de la violence politique. L'enfant, jouet pitoyable de la répression ou acteur volontaire, s'impose grâce au personnage de Gavroche. Concurremment, l'espace réel des insurrections favorise la participation des enfants, devenus sujets d'histoire. Les pratiques de ces derniers dessinent bien un monde à part, et pourtant l'action enfantine reste subordonnée aux logiques des adultes. Dans le cadre de la guerre civile, la violence devient légitime, l'engagement des enfants apparaît donc comme un rite de passage, comme une manière d'accomplir des prouesses individuelles et comme un acte de solidarité nécessaire avec les générations plus âgées. Il n'y a donc pas autonomie des ambitions, puisque lors des grèves ou des défilés, les membres de la famille partagent les mêmes aspirations. En revanche, les pratiques distinguent les différents stades de la vie. Les enfants mettent un point d'honneur, par défi et esprit ludique, à s'exposer davantage. A partir de ces années, le thème de l'enfance innocente s'évanouit, supplanté par celui de l'enfance coupable. Or, tandis que les révolutions s'achèvent, la seule manière de pacifier la société, disent les politiques habiles, c'est d'éduquer les enfants des «classes laborieuses et vicieuses ». Ce n'est pas tout à fait un hasard si dès le mois de mai 1871 Le National raille férocement les gamins contribuant à l'érection des barricades, « Ils mettent, dit-il, à cette occupation certainement plus d'entrain que s'ils étaient à l'école obligés de faire leur leçon " ${ }^{40}$. Il n'est pas non plus anodin de constater que le 2 juin, La Patrie revient à la charge. Mais pour la rédaction du journal, la contribution apportée à la construction est moins blâmable que l'oisiveté et le «jeu en tête ». On n'en verra plus, affirme-t-il joyeusement, " par centaines dans certaines rues et dans certains carrefours pour y jouer au bouchon ou à pile ou face " ${ }^{41}$. Ils pourront donc reprendre le "chemin de l'école ou de l'atelier ». Le XIXe siècle a donc forgé, de 1832 à 1871, à l'ombre des révolutions, l'archétype du gamin des barricades. 


\section{NOTES}

1. . Les Misérables, L.G.F., 1985. Rappelons que le récit de la mort de Gavroche se situe en 1832, à la suite de l'insurrection provoquée par l'enterrement du général Lamarque. 2. Larousse (Pierre), Grand Dictionnaire universel du XIXe siècle, tome septième, Paris, 1870, p. 535.

3. Les dixneuvièmistes ont peu étudié jusqu'à présent la place de l'enfant dans la société, dans la mesure où ceux qui s'attachent à l'étude des âges de la vie ont privilégié des "groupes" plus amples : la vieillesse ou la jeunesse. Cf. sur ce dernier point l'excellent numéro de 1848, Révolutions et mutations au XIXe siècle, $n^{\circ} 8$, "Jeunesses au XIXe siècle”, 1992. Cf. aussi les travaux de Jean-Noël Luc sur la petite enfance.

4. Cf. en particulier les études sur la loi du 21 mars 1841 limitant le travail des enfants dans l'industrie.

5. Perrot (Michèle) dans Les Ouvriers en grève (France 1871-1890), Paris, Mouton, 1974, 2 vol. ; ou Lequin (Yves), dans Histoire des Français (XIXe-XXe siècle), 2. La Société, Paris, A. Colin, 1983, n'ont pas noté de comportements spécifiques à l'enfance.

6. Faure (Alain), "Enfance ouvrière, enfance coupable", Les Révoltes logiques, $\mathrm{n}^{\circ} 13$, 1981, pp. 13-35.

7. Cf. les travaux en cours de Pierre (Eric) qui a composé la bibliographie complémentaire du livre classique de Gaillac (Henri), Les Maisons de correction (1830-1945), Paris, Editions Cujas, 1991 (1970).

8. Cf. Zeldin (Théodore), Histoire des passions françaises, 1848-1945, I. Ambition et amour, chap. 12, Les enfants, Paris, Recherches, 1978.

9. Cf. Mayeur (Françoise), Histoire générale de l'enseignement et de l'éducation en France, T.3, De la Révolution à l'école républicaine, Paris, Nouv. lib. de France, 1981. 10. Crubellier (Maurice), L'Ecole Républicaine, Editions Christian, 1993, pp. 115-128, et du même auteur l'ouvrage devenu classique L'enfance et la jeunesse dans la société française, 1800-1950, Paris, A. Colin, Coll. U., 1979.

11. Ténot (E.), cité par Vigier (Philippe), La vie quotidienne en province et à Paris pendant les journées de 1848, Paris, Hachette, 1982, p. 311.

12. Expression empruntée à Girard (Louis), Napoléon III, Paris, Fayard, 1986.

13. Lissagaray (P.O.), Histoire de la Commune de Paris, Paris, 1972 (1896), Maspéro, p. 232.

14. La Patrie du 2 juin 1871.

15. Grand Dictionnaire universel du XIXe siècle, Larousse (Pierre), tome onzième, Paris, 1874, pp. 332-333.

16. Cf. Girardet (Raoul), Mythes et mythologies politiques, Paris, Seuil, 1986, voir en particulier l'introduction et la conclusion en forme d'essai d'interprétation.

17. Cf. Lejeune (Dr), Faut-il fouetter les "apaches" ?..., Paris, Librairie du Temple, 1910.

18. Ariès (Philippe), L'enfant et la vie familiale sous l'Ancien Régime, Paris, Seuil (1963) 1973, p. 51.

19. Weill (George), Histoire du parti républicain en France (1814-1870), Slatkine (réimpression de l'édition de 1928), Genève, 1980, pp. 7-8.

20. Montfalcon (Jean-Baptiste), Histoire des insurrections de Lyon..., citée par Rude (Fernand), Les révoltes des canuts (1831-1834), Paris, Maspéro, 1980, p. 41. 
21. Dumas (Alexandre), Mes Mémoires (1830-1833), Paris, Robert Laffont, Coll. Bouquins, 1989, p. 835, les propos d'Alexandre Dumas sont recoupé en partie par Noël Parfait et Louis Blanc.

22. Hugo (Victor), Choses vues, 1847-1848, Paris, Gallimard, 1972, p. 296.

23. Idem., p. 319.

24. AN. BB/30/358.

25. Cf. par exemple L'évolution intellectuelle et morale de l'enfant de G. Compayré, cité par Zeldin (Theodore), Histoire des passions françaises (1848-1945), I. Ambition et amour, Paris, Seuil, 1980 (1978), p. 377.

26. La Gazette des tribunaux du 1er février 1840.

27. Le siècle du 27 mai 1871.

28. La Patrie du 28 mai relatant la journée du 23 mai.

29. Lissagaray (P.-O.), Histoire de la Commune de Paris, op. cit., p. 336.

30. Victorine B., Souvenirs d'une morte vivante, Paris, Maspéro, 1976 (1909), p. 213.

31. Idem., p. 353.

32. L'Officiel du 24 mai 1871.

33. Le National du 27 mai 1871.

34. Lissagaray (P.-O.), Histoire de la Commune de paris, op. cit., p. 353.

35. Le Procès de la Commune. Compte rendu des débats du conseil de guerre...deuxième série, 21e livraison, Affaire des pupilles de la Commune, pp. 162-164, citée par Jacques Rougerie, Procès des Communards, Gallimard/Julliard, coll. Archives, 1978, p. 122-123. 36. Lissagaray (P.-O.), Histoire de la Commune de paris, op. cit., p. 352.

37. Enquête parlementaire sur l'insurrection du 18 mars, pp. 549-550. Rapport du colonel Gaillard† : Du rôle des enfants dans l'insurrection, cité par Rougerie (Jacques), op. cit., pp. 120-121.

38. Le siècle du 27 mai 1871.

39. Le Siècle du 2 juin 1871.

40. Le National du 27 mai 1871.

41. La Patrie du 2 juin 1871.

\section{RÉSUMÉS}

L'historiographie du XIXe siècle s'est peu intéressée à l'histoire des enfants, et encore moins à celle de la violence politique du groupe enfantin. Jusqu'à présent l'évocation de Gavroche suffisait à l'explication. Or il convient de se demander comment la représentation du massacre d'un jeune garçon, à laquelle toute violence juvénile fait encore référence s'est imposée. Retrouver la manière dont s'est construit l'archétype, le passage d'un héros de roman à celui du coeur, dont témoigne entre autre la dernière livraison du Grand dictionnaire universel du XIXe siècle de Pierre Larousse, est une étape obligée qui doit cependant être confrontée à " la consistance du réel ». A Lyon, lors la révolte des Canuts, à Paris au moment des barricades juin 1832, en février et juin 1848 des témoignages variés signalent la présence d'enfants. Mais, c'est avec la guerre des barricades, en mai 1871, que la participation massive des enfants, d'abord comme terrassiers s'attachant aux travaux de fortification puis comme combattants, est attestée. 
Et pourtant si la violence devient parfois un rite de passage obligé, l'action enfantine reste subordonnée aux logiques des adultes.

INDEX

Index géographique : France

Index chronologique : 19e siècle

Mots-clés : histoire, enfants, violence

\section{AUTEUR}

\section{FRÉDÉRIC CHAUVAUD}

Maître de conférences à l'Université de Poitiers. Il a publié récemment Les passions villageoises au XIXe siècle, Publisud, 1995. Ses recherches actuelles portent sur l'histoire de l'organisation judiciaire, Le juge le tribun et le comptable, Anthropos-Economica, 1995. 